\title{
Kounis Syndrome in a Clinical Case
}

\section{Síndrome de Kounis a Propósito de un Caso Clínico}

\author{
Ángel Javier Peñafiel Chávez ${ }^{1}$, Alpha Baba Dioubate² ${ }^{2}$ Jessica Magali Herrera \\ Abarca $^{1}$, and Mauricio Tomas Rivas Contreras ${ }^{3}$ \\ 1Departamento de Medicina, Facultad de Salud Pública, Escuela Superior Politécnica de \\ Chimborazo, Riobamba, Ecuador \\ ${ }^{2}$ Departamento Médico, Centro de Especialidades Médicas CARDIOPIEL, Riobamba, Ecuador \\ ${ }^{3}$ Departamento Médico, Clínica Dermatológica del Norte, Ovalle, Chile
}

XVIII International Seminar on Health, Food and Human Nutrition

Corresponding Author:

Ángel Javier Peñafiel Chávez angel.peniafiel@espoch.edu.ec

Published: 9 September 2021

Production and Hosting by Knowledge E

(c) Ángel Javier Peñafiel Chávez et al. This article is distributed under the terms of the Creative Commons Attribution License, which permits unrestricted use and redistribution provided that the original author and source are credited.

\section{Abstract}

Kounis syndrome is the grouping of an acute coronary artery disease associated with an anaphylactic allergy, in which there is an antigen-antibody reaction caused by the release of inflammatory mediators and mast cells act by causing degranulation, and in this situation there is the presence of cells of inflammation, causing an anaphylactic reaction. There are several triggers that can trigger an allergic reaction and therefore Kounis syndrome, patients with atopy are more susceptible, it has been associated with insect bites, medications and some foods, environmental contamination and some medical conditions. Any age because it has been described even in children. There are not many clinical studies, nor a larger sample of patients to reach a consensus on this pathology. We present a 46-year-old patient who is admitted to the Emergency service of the national institute of cardiology and cardiovascular surgery in Havana. Cuba; with a history of Systemic Arterial Hypertension (HT), which is an increase in blood pressure above the upper limits of normality. Grade II obesity, which is a body mass index $\geq 35$. In addition to referred dyslipidemia. In the results according to the diagnostic criteria of Kounis syndrome, the following were found: Signs and symptoms of myocardial ischemia, urticaria, pruritus, dyspnea, sinus tachycardia after bee sting. Electrocardiogram (ECG): With ST segment elevation in 2 or more continuous leads. Echocardiography: Transient segmental motility disorders. Cardiac chambers of preserved size and function. Serum myocardial biomarkers within normal parameters, increased Histamine, Tryptase, leukotriene values. The objective is to describe the clinical characteristics, complementary examinations, their diagnosis, evolution and treatment. It was concluded that the diagnosis of Kounis syndrome is eminently clinical. in itself everything that leads to the activation of mast cells can produce the syndrome. The prognosis depends on the type of Kounis, cardiovascular risk factors and pre-existing coronary artery disease.

Keywords: ischemia, inflammatory response, angina, vasospasm, atherosclerosis, histamine.

\section{Resumen}

El síndrome de Kounis es la agrupación de una arteriopatía coronaria aguda asociado a una alergia anafiláctica, en la que hay una reacción antígeno-anticuerpo causado por la liberación de mediadores inflamatorios los mastocitos y estos actúan provocando degranulación, y ante esta situación hay presencia de células de la inflamación, provocando una reacción anafiláctica. Existen varios gatillantes que pueden desencadenar una reacción alérgica y por ende el síndrome de Kounis, son más susceptibles los pacientes con atopia, se le ha relacionado mucho con picaduras de insectos, medicamentos y algunos alimentos, contaminación ambiental y 
algunas condiciones médicas Se puede presentar a cualquier edad porque se ha descrito incluso en niños. No hay muchos estudios clínicos, ni una muestra más grande de pacientes para realizar un consenso de esta patología. Presentamos una paciente de 46 años de edad que es ingresada al servicio de Emergencia del instituto nacional de cardiología y cirugía cardiovascular de la habana. Cuba; con antecedente de Hipertensión Arterial Sistémica (HTA) que es aumento de la presión arterial por encima de los limites superiores de normalidad. Obesidad grado II que es el índice de masa corporal $\geq 35$. además de dislipidemia referida. En los resultados según los criterios diagnóstico del síndrome de Kounis se encontró: Signos y síntomas de isquémica miocárdica, urticaria, prurito, disnea, taquicardia sinusal tras picadura de una abeja. Electrocardiograma (ECG): Con elevación del segmento ST en 2 o más derivaciones continuas. Ecocardiografía: Trastornos de motilidad segmentaria transitorio. cavidades cardiacas de tamaño y función conservada. Los biomarcadores miocárdicos sérico dentro de los parámetros normales, incremento de los valores de Histamina, Triptasa, leucotrienos. El objetivo es describir las características clínicas, exámenes complementarios, su diagnóstico, evolución y tratamiento. Se concluyó que el diagnóstico del síndrome de Kounis es eminentemente clínico. en sí todo lo que conlleve a la activación de mastocitos puede producir el síndrome. El pronóstico depende del tipo de Kounis, de los factores de riesgos cardiovasculares y coronariopatía preexistente.

Palabras Clave: isquemia, respuesta inflamatoria, angina, vasoespasmo, ateroesclerosis, histamina.

\section{Introducción}

El síndrome de Kounis es una arteriopatía coronaria aguda asociado a una alergia anafiláctica, el cual es causado por la liberación de mediadores inflamatorios los mastocitos actúan provocando degranulación, ante esta situación hay presencia de células de la inflamación, provocando una reacción anafiláctica [1,2]. Los mediadores que participan son: Triptasa, productos del ácido araquidónico, factor activador de plaquetas y varias citoquinas y quimiocinas que se liberan en este proceso [1-3]. La presencia de reacciones alérgicas a nivel general es alrededor de 1,6\% o incluso mayor. Existen varios gatillantes que pueden desencadenar una reacción alérgica y por ende el síndrome de Kounis, son más susceptibles los pacientes con atopia [46]. El síndrome de Kounis (SK) definido en 1991 por Kounis y Zavras, abarca conceptos como una angina alérgica e infarto alérgico y se le ha relacionado mucho con picaduras de insectos, medicamentos y algunos alimentos, contaminación ambiental y algunas condiciones médicas. No se conoce su verdadera incidencia ya que, no hay muchos casos reportados [7-9].

\subsection{Conceptos fisiopatológicos}

Las células principales células en responder son los mastocitos; son lo más importante para desencadenar reacciones alérgicas. Se derivan de células madre que expresan el marcador CD 34 en la médula ósea, posteriormente por el factor de crecimiento de mastocitos se convierten en células maduras para producir reacciones alérgicas 
inmediatas, mediadas por inmunoglobulina $\mathrm{E}$; pero no es esta única molécula la que puede activar los mastocitos, también las anafilotoxinas (C3a-C5a) conducen a la liberación y degranulación de los mastocitos con la consiguiente salida de mediadores inflamatorios y vasoactivos tanto locales como sistémicos, los cuales al interactuar con otras células como macrófagos y linfocitos T producen el síndrome [10-12].

Se sabe que para que se produzca degranulación debe haber por lo menos 2.000 anticuerpos ligados a receptores en la superficie del mastocito que pueden ser de diferentes especificidades $[13,14]$. Cabe tener en cuenta que la activación mediada por IgE, es la que activa estas células. Los macrófagos activan a más macrófagos y pueden intensificar la respuesta para la activación de linfocitos $\mathrm{T}$; la proteína $1^{\text {a }}$ que es inducible en los macrófagos, puede activar más mastocitos, mientras CD 169 de los macrófagos activa linfocitos CD 8; estas células T median la activación y proliferación de mastocitos y a su vez regulan la actividad de los macrófagos $[15,16]$.

La interacción de estos dos receptores media la disminución de la presión diastólica, hecho que aumenta la presión de pulso [17]. Igualmente, induce la expresión de factor tisular y taquicardia mediada por barorreceptores al disminuir la presión aórtica media $[16,17]$. Otra célula relacionada con reacciones de hipersensibilidad e inflamación es el eosinófilo a través de su proteína catiónica eosinofílica $[16,18,19]$. Se ha demostrado que la activación de estas células al igual que la de las plaquetas, induce trombosis del stent [20-22].

El factor activador de plaquetas disminuye el flujo coronario, retrasa la conducción atrioventricular y produce efectos depresores en el miocardio [21, 23].

La betatriptasa, que es la enzima activa, es una proteasa que se encuentra en los gránulos de los mastocitos y se libera cuando estas células se degranulan, activan el complemento y las vías de coagulación [24, 25].

En un estudio en Japón se evidenció que los pacientes con angina inestable e infarto de miocardio tenían mayores niveles de triptasa que aquellos con placas de menos del 50\%, y que este marcador asociado a las quinasas son predictores de vulnerabilidad de la placa [23]. El sistema kinina-kalicreína, que produce hipotensión y coagulación intravascular diseminada en casos severos de anafilaxia, ejerce una acción dual en la cascada de coagulación dado que tiene propiedades tanto fibrinolíticas como trombóticas [22, 26, 27].

La quinasa y catepsina $D$ actúan como enzimas para convertir la angiotensina I en angiotensina II como principal sustancia vasoconstrictora [24].

El corazón puede ser el sitio primario de la reacción anafiláctica, dado que cuando esta ocurre hay vasoconstricción coronaria, fenómeno que conlleva isquemia del miocardio y finalmente disfunción ventricular, reducción del retorno venoso, salida de plasma al espacio intersticial y pérdida de volumen por permeabilidad capilar por lo cual cae el gasto cardiaco y se produce hipoperfusión coronaria y daño miocárdico consecuente [26-28]. Se ha demostrado que en el corazón de los mamíferos hay gran cantidad de mastocitos en los vasos coronarios, en la capa íntima y en las placas de ateroma. Cada vez hay mayor evidencia a favor que los mastocitos intervienen en la iniciación y progresión de la placa a través de sus mediadores como la histamina. 
Igualmente desempeñan un papel preponderante en la vulnerabilidad de la placa $[8,9,29]$.

\subsection{Etiología}

Con relación a la etiología de la entidad, se ha visto que puede ser multifactorial, e incluir reacciones alérgicas a medicamentos, condiciones médicas, exposiciones ambientales, etc.

\subsection{Abordaje diagnóstico}

El diagnóstico dl síndrome de Kounis es eminentemente clínico los enfoques diagnósticos no invasivos son cada vez más fiables y eficientes [2, 3].

Los principales síntomas son dolor torácico (el cual puede ser atípico en comparación con el que ocurre en el síndrome coronario de causa no alérgica), disnea, palpitaciones y síntomas disautonómicos [27, 29, 30].

Se pueden evidenciar diferentes patrones en el electrocardiograma. En la mayoría de los casos reportados se evidencia supradesnivel del ST en la cara anterior e inferior; igualmente puede ser normal o presentar cambios inespecíficos del segmento ST$\mathrm{T}$ [31-33] siendo por consiguiente la descendente anterior y la coronaria derecha las arterias más comprometidas. Otras anormalidades incluyen cualquier grado de bloqueo y arritmias cardiacos, principalmente fibrilación auricular, ritmo nodal y ectopias ventriculares [31-35].

\subsection{Variantes del síndrome de Kounis}

Tipo I: Arterias coronarias normales, sin factores de riesgo cardiovascular.

Tipo II: Enfermedad aterosclerótica preexistente.

Tipo III: Trombosis del stent coronario $[1,36]$.

Pueden agregarse estudios para confirmar una reacción alérgica: Niveles séricos de triptasa (vida media menor a $90 \mathrm{~min}$ ), histamina (vida media menor a $10 \mathrm{~min}$ ), inmunoglobulina E, proteínas del complemento y conteo de eosinófilos [20-36].

\subsection{Línea cronológico}

Paciente de sexo femenina de 46 años con antecedente de HTA de 3 años de evolución por lo cual toma enalapril 20 mg diario. Obesidad grado II, dislipidemia referida Acude por su propio medio a emergencia del instituto nacional de cardiología y cirugía cardiovascular de la Habana. Cuba con dolor precordial de moderada intensidad opresivo con irradiación en la espalda, disnea, sudoración fría y copiosa tras picadura de una abeja, el cual se realizó estudios pertinentes donde se diagnostica el síndrome de Kounis. Ingresa en unidad de cuidados coronario-intensivos donde permanece hospitalizado por 2 días con oxígeno 3 L, clopidogrel 75 mg. vía oral, Nitroglicerina 50 mg diluidos en 
$50 \mathrm{ml}$ de solución salina 0,9\% intravenoso (INFUSION), Diltiazem $90 \mathrm{mg}$ vía oral cada 12 hr. Hidrocortisona 500 mg diluidos en $250 \mathrm{ml}$ de solución salina 0,9\% intravenoso a durar $1 \mathrm{hr}$ seguidos de $100 \mathrm{mg}$ cada $8 \mathrm{hr}$ intravenoso difenhidramina $20 \mathrm{mg} 1$ ampolla intravenoso seguido de 1 ampolla cada $8 \mathrm{hr}$ intravenoso, Ranitidina $50 \mathrm{mg} 1 \mathrm{ampolla}$ intravenosa seguido de $50 \mathrm{mg}$ cada $12 \mathrm{hr}$ intravenoso Fluido de terapia $1000 \mathrm{ml} / 24$ hr epinefrina 0,3 mg intramuscular dosis única; posterior a eso se ingresar en sala de cuidados coronarios intermedios donde permaneció por 3 días con evolución clínica satisfactoria.

\subsection{Información del paciente}

Paciente femenina de 46 años, casada, nacida y residente en cuidad de la Habana, instrucción universitaria (abogada). Con antecedentes de HTA desde hace 3 años en tratamiento con enalapril $20 \mathrm{mg}$ vía oral diario, Obesidad tipo II, dislipidemia referida, acude por su propio medio a emergencia del Instituto Nacional de Cardiología y Cirugía Cardiovascular de la Habana Cuba con dolor precordial de moderada intensidad opresivo con irradiación en la espalda, disnea, sudoración fría y copiosa tras picadura de una abeja.

\subsection{Examen físico}

Tensión arterial: 130/80 mmHg - Frecuencia Cardiaca: 108/min - Frecuencia Respiratoria: 30/min. Peso: $75 \mathrm{~kg}$ - Talla: $165 \mathrm{~cm}$ - IMC: $35 \mathrm{~kg} / \mathrm{cm}$.

El paciente lúcido, consciente, orientado en tiempo, espacio y en persona. hidratado afebril.

Mucosa: Húmedas y normocoloreadas; tejido celular subcutáneo no infiltrado por edema; respiratorio murmullo vesicular normal, no estertores, uso de músculos accesorios respiratorios; corazón: Latido de la punta visible en el 5 to espacio intercostal, no Thrill, ruidos cardiacos audibles de buen tono, rítmicos, no soplo sistólico. P2 acentuado. pulsos periférico presente; Abdomen: Ruidos hidroaéreos presentes, blando globuloso, no doloroso a la palpación tanto superficial como profundo no Tumoración palpable.

\subsection{Evaluación diagnóstica: Complemetarios}

Hemograma Hb......... 16,9 g/L

WBC.......... 10,6 × $109 / \mathrm{L}$

PLT......... $201 \times 109 / \mathrm{L}$

Troponinas $<40$ ng (parámetro normal)

\subsection{Electrocardiograma: Durante la crisis vasoespastica}

'Se traslada a salón de hemodinámica’ (CORONARIOS NORMALES). 


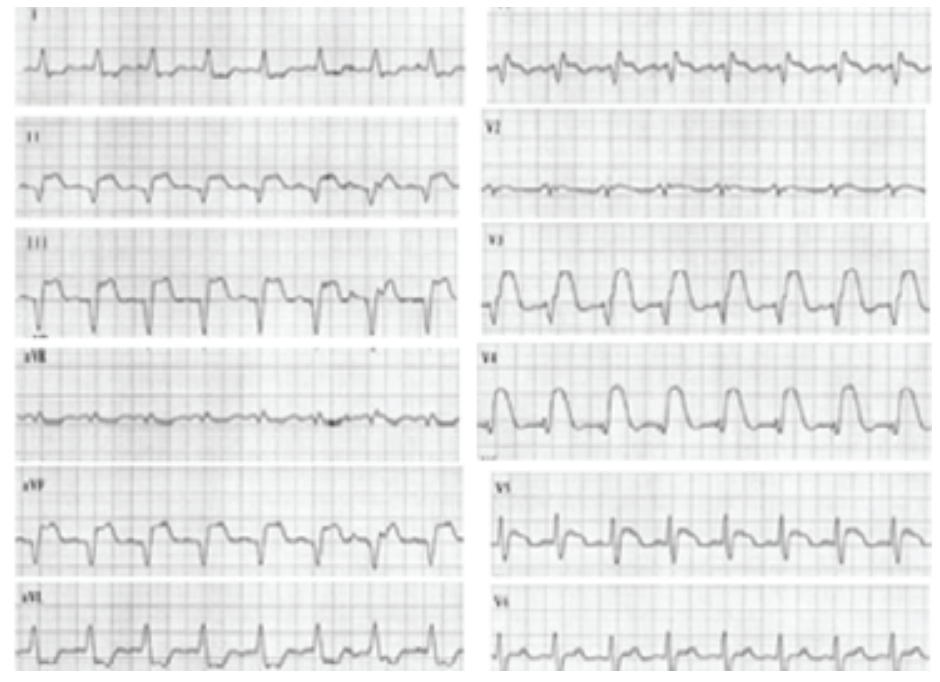

Figure 1

Electrocardiograma: Durante la crisis vasoespástica.
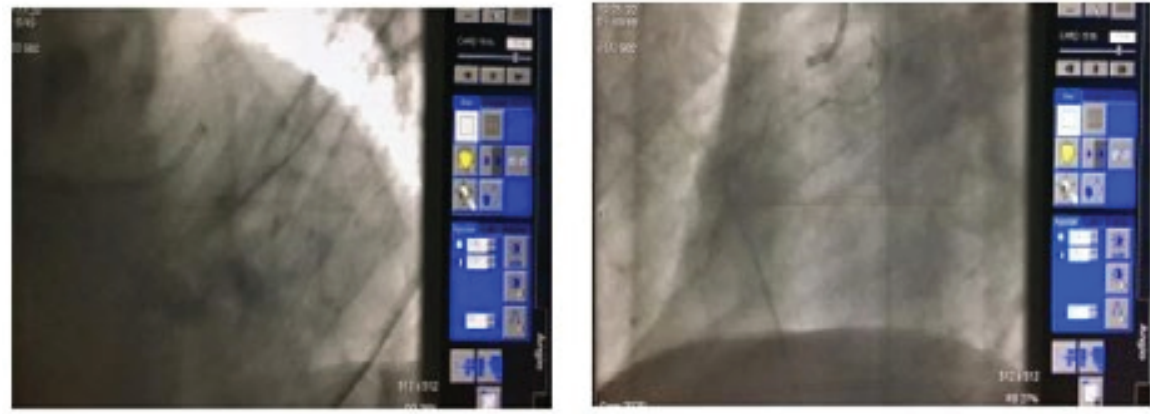

Figure 2

Gráfico 2 y 3: Coronariografía normal de ambas arterias coronarias.

\subsection{Conducta terapéutica}

Ingresa en unidad de cuidados coronario-intensivos donde permanece hospitalizado por 2 días con oxígeno $3 \mathrm{~L}$, clopidogrel $75 \mathrm{mg}$ vía oral, Nitroglicerina $50 \mathrm{mg}$ diluidos en $50 \mathrm{ml}$ de solución salina 0,9\% intravenosa (INFUSIÓN), Diltiazem $90 \mathrm{mg}$ vía oral cada $12 \mathrm{hr}$. Hidrocortisona $500 \mathrm{mg}$ diluidos en $250 \mathrm{ml}$ de solución salina 0,9\% intravenosa a durar $1 \mathrm{hr}$ seguidos de $100 \mathrm{mg}$ cada $8 \mathrm{hr}$ intravenosa, difenhidramina $20 \mathrm{mg} 1$ ampolla intravenosa seguido de 1 ampolla cada $8 \mathrm{hr}$ intravenosa, Ranitidina $50 \mathrm{mg} 1 \mathrm{ampolla}$ intravenosa seguido de $50 \mathrm{mg}$ cada $12 \mathrm{hr}$ intravenosa. Fluido de terapia $1000 \mathrm{ml} / 24$ hr Epinefrina 0,3 mg intramuscular dosis única; posterior a eso se ingresar en sala de cuidados coronarios intermedios donde permaneció por 3 días con evolución clínica satisfactoria. 


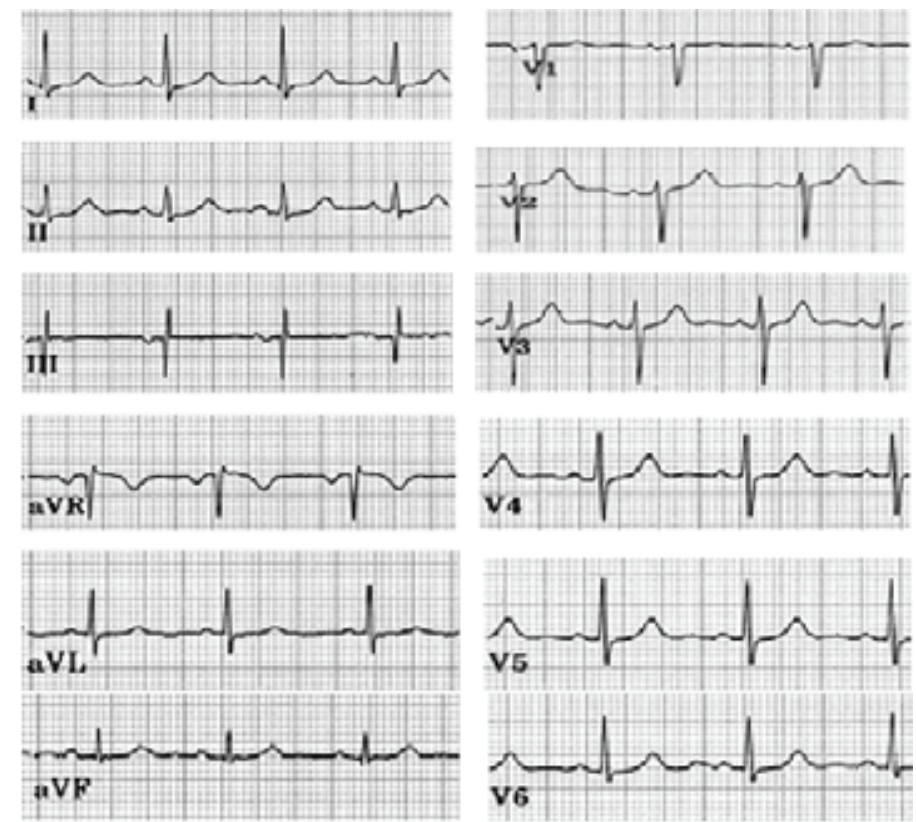

Figure 3

Electrocardiograma normal.

\subsection{EKG: Evolutivo (normal)}

Ecocardiografía Transtorácico: (ETT EVOLUTIVO).

- Buena función sistólica biventricular en reposo.

- Contractilidad global y segmentaria conservada del VI y VD en reposo.

- Aparatos valvulares competentes-No masas, no trombos, no derrames.

- No Hipertensión Pulmonar.

\subsection{Seguimiento y respuesta terapéutica}

Es valorado por distintas especialidades (Alergólogos, medicina interna, cardiología) quienes confirma el diagnóstico y recomiendan continuar con tratamiento correspondiente. La paciente con buena perspectiva sobre su enfermedad.

\section{Discusión}

El paciente de 46 años de edad con antecedentes de HTA, obesidad, dislipidemia referida con el diagnóstico de síndrome de Kounis cuyo diagnóstico es eminentemente clínico en el cual los criterios diagnósticos mencionados anteriormente coinciden con bibliografías internacionales por lo que concluimos que los criterios clínicos, electrocardiograma más los medios diagnósticos de laboratorios constituyen los bases fundamentales para el diagnóstico, y la fluidoterapia, corticoesteroides, anticálcicos, vasodilatadores, antihistamínicos, epinefrina además de fármacos para el tratamiento 
del síndrome coronario aguda en dependencia de si existe o no lesión coronaria significativa, representan los principales pilares del tratamiento del síndrome de kounis [1-36].

\subsection{Perspectiva del paciente}

La paciente describe a su problema de salud como una situación de mucho estrés incluso pensó en que iba a morir, pero se sentía con confianza ya que se encontraba en un Instituto de Cardiología y día a día ella puso empeño en tener buen ánimo para ir mejorando.

\subsection{Consentimiento informado}

Se cuenta con el consentimiento informado y firmado por la paciente.

\section{Conclusión}

Se considera que se deben realizar más estudios para conocer la epidemiología, diagnóstico y tratamiento óptimo de un síndrome que pasados 20 años de su descripción inicial continúa siendo un gran desconocido.

\section{Conflicto de Intereses}

Este trabajo cumple con los requisitos sobre consentimiento informado, y sobre la ausencia de conflictos de intereses

según corresponda.

\section{Limitación de Responsabilidad}

Nosotros los autores declaramos que todos los puntos de vista expresados en este trabajo son de nuestra entera responsabilidad.

\section{Fuentes de Apoyo}

Este trabajo fue realizado con la autofinanciación de los autores.

\section{References}

[1] Arora S, Patel R, Fadila M, Wool K. The atopic heart: A curious case of coronary hypersensitivity. Neth J Med. 2016;74:130-132.

[2] Fourie P. Kounis syndrome: A narrative review. South Afr J Anaesth Analg. 2016;22:72-80.

[3] López-Abad R, Rodríguez F, García-Abujeta JL, Martín-Gil D, Jerez J. Myocardial ischemia due to severe amoxicillin allergy. J Investig Allergol Clin Immunol. 2004;14(2):162-4. 
[4] Alevizos M, Karagkouni A, Panagiotidou S, Vasiadi M, Theoharides RC. Stress triggers coronary mast cells leading to cardiac events. Ann Allergy Asthma Immunol. 2014;112:309-316.

[5] Soufras GD, Lianas D, Patsouras N, Tsigkas G, Kounis NG. Kounis syndrome: Aspects on pathophysiology and management. Eur J Intern Med. 2016;32:e30-e31.

[6] Kumara-Ralapanawa DMP, Kularatne SAM. Kounis syndrome secondary to amoxicillin/clavulanic acid administration: A case report and review of literature. BMC Res Notes. 2015;8:97.

[7] Ridella M, Bagdure S, Nugent K, Cevik C. Kounis syndrome following beta-lactam antibiotic use: Review of literature. Inflammation \& Allergy- Drug Targets. 8:11-16.

[8] Kounis NG, Soufras GD, Davlouros P, Tsigkas G, Hahalis G. Combined etiology of anaphylactic cardiogenic shock: Amiodarone, epinephrine, cardioverter defibrillator, left ventricular assist devices and the Kounis syndrome. Ann Cardiac Anaesthesia. 2015;8:261-264.

[9] Kounis NG, Soufras GD, Tsigkas G, Hahalis G. White blood cell counts, leukocyte ratios, and eosinophils as inflammatory markers in patients with coronary artery disease. Clin Appl Thromb Hemost. 2015;21:139-143.

[10] Chen JP, Hou D, Pendyala L, Goudevenos JA, Kounis NG. Drug-eluting stent thrombosis: The Kounis hypersensitivity-associated acute coronary syndrome revisited. JACC Cardiovasc Interv. 2009.2:583593.

[11] Yanagawa Y, Nishi K, Tomiharu N, Kawaguchi T. A case of Tako-tsubo cardiomyopathy associated with Kounis syndrome. Int J Cardiol. 2009;132:e65-e67.

[12] Dippenaar JM, Naidoo S. Allergic reactions and anaphylaxis during anaesthesia. Current allergy \& clinical Immunology. 2015;28(1).

[13] Kounis G, Soufras G, Hahalis. Anaphylactic shock: Kounis hypersensitivity associated syndrome seems to be the primary cause. N Am J Med Sci. 2013;5:31-36.

[14] Nicholas G, Kounis S, Giannopoulos GD, Soufras GN, Kounis J. Goudevenos. Foods, drugs and environmental factors: Novel Kounis syndrome offenders. Intern Med. 2015;54:1577-1582.

[15] Renda F, Landoni G, Trotta F, Piras D, Finco G, Felicetti P. Kounis syndrome: An analysis of spontaneous reports from international pharmacovigilance database. Inter J Cardiol. 2016;21:7-20.

[16] Almpanis G, Siahos S, Karogiannis NC, et al. Kounis syndrome: Two extraordinary cases. Int J Cardiol. 2011;147:e35-e38.

[17] Lieberman P, Simons FE. Anaphylaxis and cardiovascular disease: Therapeutic dilemmas. Clinical \& Experimental Allergy. 45(8):1365-2222.

[18] Patanè S, Marte F, Di Bella G, Chiofalo S, Currò AS. Coglitore. Acute myocardial infarctiona and Kounis syndrome. Int J Cardiol, 2009;134:e45-e46.

[19] Chen JP, Hou D, Pendyala L, Goudevenos JA, Kounis NG. Drug-eluting stent thrombosis: The Kounis hypersensitivity-associated acute coronary syndrome revisited. JACC Cardiovasc Interv. 2009;2:583593.

[20] Kounis NG, Hahalis G, Kounis SA, Kounis GN. Kounis syndrome and simultaneous multivessel acute coronary syndromes after successful drug-eluting stent implantation. Int J Cardiol. 2008;127:146-148.

[21] Venturini E, Magni L, Kounis NG. Drug eluting stent-induced Kounis syndrome. Int J Cardiol. 2011;146:e16e98.

[22] Patanè S, Marte F, Currò A, Cimino C. Recurrent acute myocardial infarction and Kounis syndrome. Int J Cardiol. 2010;142:e20-e22.

[23] Vivas D, Rubira JC, Ortiz AF, Macaya C. Coronary spasm and hypersensitivity to amoxicilin: Kounis or not Kounis syndrome? Int J Cardiol. 2008;128:279-281.

[24] Kounis GN, Kounis SA, Hahalis G, Kounis NG. Coronary artery spasm associated with eosinophilia: Another manifestation of Kounis syndrome? Heart lung Circ. 2009;18:163-164.

[25] Yong SC, Hyun K, Min HB, et al. Evaluation of myocardial injury through serum troponin I and echocardiography in anaphylaxis. A J Emerg Med. 2016;34:140-144.

[26] Dippenaar JM, Naidoo S. Allergic reactions and anaphylaxis during anaesthesia. Current Allergy \& Clinical Immunology. 2015;28(1).

[27] Doğan V, Mert GO. syndrome. Int J Cardiol. 2009;134:e129-e131.

[28] Biteker FS, Mert KU, Biteker M. Treatment of Kounis syndrome. I J Cardiol. 2015;181:133-134.

[29] Baldomà N, Cosmen CR, Galinski SF, García LM, Gracia LR, Villén FE. Serum tryptase levels in acute coronary syndromes with ST elevation. Int J Cardiol. 2009;131:403-404.

[30] Wong CW, Luis S, Zeng I, Stewart RA. Eosinophilia and coronary artery vasospasm. Heart Lung Circ. 2008;17:488-496.

[31] Cevik C, Nugent K, Shome GP, Kounis NG.Treatment of Kounis syndrome. Int J Cardiol. 2010;143:223226 
[32] Pampín F, Rial Prado MJ, Vázquez Vigo R, González Guzmán LA. Síndrome coronario agudo por hipersensibilidad: Síndrome de Kounis. Galicia Clin. 75(1):31-32.

[33] López-Abad R, Rodríguez F, García-Abujeta JL, Martín-Gil D, Jerez J. Myocardial ischemia due to severe amoxicillin allergy. J Investig Allergol Clin Immunol. 2004;14(2):162-4.

[34] Lin RY, Curry A, Pesola GR, et al. Improved outcomes in patients with acute allergic syndromes who are treated with combined $\mathrm{H} 1$ and $\mathrm{H} 2$ antagonist. Ann Emerg Med. 2000;36:462-468.

[35] Franco AJD, Doblas JJG, García JMH, et al. Treatment of refractory vasoespastic angina with corticosteroids. A case report. Int J Cardiol. 2007;118:e51-e53.

[36] Takagi S, Goto Y, Hirose E, et al. Successful treatment of refractory vasospastic angina with corticosteroids: Coronary arterial hyperactivity caused by local inflammation. Circ J. 2004;68: 17-22.

[37] Cepeda PR, Herrejón EP, Aguirregabiria MMR. Síndrome de Kounis. Med Intensiva. 36(5):358-364.

[38] Biteker. Current understanding of Kounis syndrome. Expert Rev Clin Inmunol. 2010;6:777-788. 